\title{
An Experimental View on Committees Providing Justified Representation
}

\author{
Robert Bredereck $^{1}$, Piotr Faliszewski ${ }^{2}$, Andrzej Kaczmarczyk ${ }^{1}$ and Rolf Niedermeier ${ }^{1}$ \\ ${ }^{1}$ TU Berlin, Berlin, Germany \\ ${ }^{2}$ AGH University, Kraków, Poland \\ robert.bredereck@tu-berlin.de, faliszew@agh.edu.pl, \{a.kaczmarczyk, rolf.niedermeier\}@tu-berlin.de
}

\begin{abstract}
We provide an experimental study of committees that achieve (proportional/extended) justified representation (JR/PJR/EJR). In particular, we ask how many such committees exist and how varied they are in terms of voter satisfaction and coverage. We find that under many natural distributions of preferences a large fraction of randomly selected JR committees also provide PJR and EJR. Further, we find that the sets of JR committees for our elections are very varied and include both high-quality ones and not-so-appealing ones.
\end{abstract}

\section{Introduction}

There exist many situations where societies-either natural or artificial-need to select a group of candidates that would represent them in some appealing way. For example, faculty members need to select their representatives in the university's senate, web search engines need to select answers to given queries that represent various interpretations [Skowron et al., 2017], and funding committees need to select projects that represent different areas of research (in addition to the excellence criterion, of course). Formally, such problems are studied under the umbrella of multiwinner voting and have recently received increased interest (see the overview of Faliszewski et al. [2017b]).

We focus on approval elections, where each voter indicates by which candidates he or she feels represented. This is a particularly convenient model because approval ballots have a simple interpretation and appear to be sufficiently expressive for many applications. Further, the notions of justified representation (JR) [Aziz et al., 2017], as well as its more constrained variants such as proportional justified representation (PJR) [Sánchez-Fernández et al., 2017] and extended justified representation (EJR) [Aziz et al., 2017], provide convincing formalizations of the idea that a committee is representative (or proportional). Briefly put, for an election with $n$ voters, a committee of size $k$ provides justified representation if there is no group of $n / k$ voters such that all these voters approve some common candidate, but none of the voters approves any member of the committee (the rationale here is that a group of $n / k$ voters is large enough to convincingly argue that its members deserve representation). Proportional justified representation and extended justified representation add similar conditions for larger groups of voters, with more commonly approved candidates.

The notions of (proportional/extended) justified representation received significant attention from the research community and, for example, it is known that JR/PJR/EJR committees always exist and that a number of voting rules, both natural and specifically designed for this purpose, provide such committees [Aziz et al., 2017; Aziz et al., 2018; Sánchez-Fernández et al., 2017; Brill et al., 2017a] (see also the overview of Elkind [2017]). Yet, so far, all these studies focused on theoretical results and experimental evaluations were missing. We believe that it is important to fill this gap and our goal is to initiate experimental investigations into the nature of JR, PJR, and EJR committees.

One of the most basic questions regarding JR, PJR, and EJR is how restrictive these notions are. On the formal level, we know that the EJR definition is more demanding than the PJR one, which in turn is more demanding than the JR one, but we are interested in a more quantitative perspective. Specifically, for a given family of elections (e.g., those generated according to the impartial culture model or one of the Euclidean models), we would like to know the average number of committees that provide (proportional/extended) justified representation. Unfortunately, we show that computing such a number is \#P-hard. Thus, for the families of elections that we consider, we draw committees uniformly at random and check how many of them satisfy JR, PJR, and EJR. Rather surprisingly, it turns out that the number of thus-generated JR committees that also satisfy PJR or even EJR is very high; in all of our experiments this was true for more than $50 \%$ of the JR committees, and in some settings this number was approaching $100 \%$. This is even more striking as the probability that a randomly generated committee satisfies JR is very high (even in the most demanding setting this probability was still above 14\%). These results stand in sharp contrast with the intuition that one might get from theoretical studies, which suggest that EJR is much more challenging to satisfy than JR (in particular, the polynomial-time algorithm for computing a JR committee is very simple, but corresponding algorithms for PJR and EJR require more involved ideas and analyses [Aziz et al., 2017; Sánchez-Fernández et al., 2017; Aziz et al., 2018]). 
Given the above discussion, we are interested in two issues. First, we would like to understand why so many JR committees appear in our experiments, and, second, we would like to know how varied these JR committees are. To deal with the first issue, we seek the smallest sets of candidates that already satisfy the JR definition for a given committee size; we refer to such sets as $n / k$-justifying groups. It turns out that for the families of elections that we consider, $n / k$-justifying groups are typically quite small and include fewer than half of the allowed committee members. As a consequence, there are many JR committees because to obtain one, it suffices to take a (small) $n / k$-justifying set and complement it with arbitrary candidates. On a more theoretical side, we show that every candidate who is approved by at least one voter must belong to some JR committee (that is, every candidate can be dubbed a possible JR member; nonetheless, testing if a candidate is a necessary JR member, i.e., if he or she belongs to all JR committees, is coNP-hard).

By the above discussion, we see that the constraints put on JR committees may be quite loose and, as a consequence, two JR committees may have very different properties. To verify this supposition, we consider two basic measures of quality of a (representative) committee: the number of voters that approve at least one committee member (referred to as the coverage of the committee; if a voter approves some committee member then we may say that his or her views are represented) and the average number of committee members that a voter approves (referred to as the voter satisfaction). Inspired by Lackner and Skowron [2019], for a given election we investigate experimentally what coverage and satisfaction values can be achieved by (a) arbitrary committees, (b) JR committees, and (c) committees provided by several prominent voting rules. It turns out that for our families of elections there are both JR committees of very high quality (in terms of both our criteria) and of relatively low one, but natural voting rules tend to select the high-quality ones.

All in all, we believe that taking an experimental approach shows a new perspective on the notions of JR, PJR, and EJR, and provides some new intuitions. However, our results also raise some concerns, of which the most intriguing one is whether the election families that we used truly model representative elections. We return to this issue in the final section. We omit many of the proofs and the details of the integer linear programs used due to space constraints.

\section{Preliminaries}

For a positive integer $t$, we write $[t]$ to denote the set $\{1, \ldots, t\}$. We model an election as a pair $E=(C, N)$, where $C=\left\{c_{1}, \ldots, c_{m}\right\}$ is a set of candidates and $N=[n]$ is a set of voters. Each voter $i \in N$ has approval ballot $A_{i} \subseteq C$ and we say that this voter approves candidate $c_{j} \in C$ if $c_{j} \in A_{i}$; we say that $i$ disapproves $c_{j}$ otherwise. For a candidate $c_{j} \in C$, by $N_{c_{j}}$ we mean the set of voters that approve $c_{j}$. A committee is a subset of candidates of a given size. A multiwinner voting rule $\mathcal{R}$ is a function that, given an election $E$ and committee size $k$, outputs the family of size- $k$ committees that win the election (according to this rule); we disregard the issue of tie-breaking.

\section{Voting Rules}

Consider an election $E=(C, N)$ and a committee size $k$. The Multiwinner Approval Voting (AV), the ChamberlinCourant (CC) [Chamberlin and Courant, 1983; Procaccia et al., 2008], and the Proportional Approval Voting (PAV) [Thiele, 1895; Kilgour, 2010] rules output size- $k$ committees $W$ that maximize the scores:

$$
\begin{aligned}
\operatorname{score}_{\mathrm{AV}}(W) & =\sum_{c \in W}\left|N_{c}\right|, \\
\text { score }_{\mathrm{CC}}(W) & =\left|\bigcup_{c \in W} N_{c}\right|, \\
\operatorname{score}_{\mathrm{PAV}}(W) & =\sum_{i \in N} \sum_{j=1}^{\left|W \cap A_{i}\right|} 1 / j .
\end{aligned}
$$

The AV score gives the total number of approvals that members of the committee receive, and we sometimes refer to this value as voter satisfaction. The CC score gives the number of voters that approve at least one committee member. Such voters can be seen as represented in the committee and we sometimes refer to the $\mathrm{CC}$ score as voter coverage. The PAV score is defined in a less intuitive way, but it can be shown to ensure proportional representation of the voters (see the works of Brill et al. [2017b] and Skowron and Lackner [2018]).

There is a simple polynomial-time algorithm for AV, but deciding whether there is a CC or PAV committee with a given score is NP-hard (see the works of Procaccia et al. [2008], Skowron et al. [2016] and Aziz et al. [2015]); yet there are approximation algorithms for these rules [Lu and Boutilier, 2011; Skowron et al., 2015; Byrka et al., 2018].

\section{Justified Representation}

Aziz et al. [2017] introduced the notion of justified representation (JR) to capture the basic requirements for representative committees. They also introduced extended justified representation (EJR) and, a bit later, Sánchez-Fernández et al. [2017] defined proportional justified representation (PJR). Aziz et al. [2017] also showed that for every election a JR (PJR, EJR) committee always exists.

Definition 1. Let $E=(C, N)$ be an election with $n$ voters and committee size $k$. For each positive integer $\ell$, a group $N^{\prime}$ of voters is $\ell$-large if it contains at least $\ell n / k$ voters, and it is $\ell$-cohesive if $\left|\bigcap_{i \in N^{\prime}} A_{i}\right| \geq \ell$, that is, if its members approve at least $\ell$ common candidates. Let $W$ be a size- $k$ committee:

1. $W$ provides justified representation for $E$ if for each 1large, 1-cohesive group $N^{\prime} \subseteq N$ of voters, at least one member of $N^{\prime}$ approves at least one candidate from $W$.

2. $W$ provides proportional justified representation if for each $\ell \in[k]$ and each $\ell$-large, $\ell$-cohesive group $N^{\prime} \subseteq$ $N$ of voters, we have that $\left|W \cap\left(\bigcup_{i \in N^{\prime}} A_{i}\right)\right| \geq \ell$ (i.e., at least $\ell$ members of $W$ are approved by some voters from $N^{\prime}$ ).

3. $W$ provides extended justified representation if for each $\ell \in[k]$ and each $\ell$-large, $\ell$-cohesive group $N^{\prime} \subseteq N$ of voters, there is an $i \in N^{\prime}$ such that $\left|A_{i} \cap W\right| \geq \ell$ (i.e., at least one voter in $N^{\prime}$ approves at least $\ell$ members of $W$ ).

We say that a voting rule satisfies $J R(P J R, E J R)$ if for each election and committee size it outputs a committee that provides JR (PJR, EJR). If a rule satisfies EJR, then it also satisfies PJR, and if it satisfies PJR, then it satisfies 
JR. Aziz et al. [2017] showed that the AV rule fails JR, but the CC rule satisfies it, and the PAV rule even satisfies EJR. Brill et al. [2017a] showed, for example, that the sequential Phragmén rule satisfies PJR.

There is a simple polynomial-time algorithm for verifying whether a given committee provides justified representation [Aziz et al., 2017], but the same task is coNP-complete for both PJR and EJR [Aziz et al., 2018; Aziz et al., 2017].

\section{Experimental Setup}

We focus on elections generated according to (a variant of) the impartial culture model and two Euclidean models [Enelow and Hinich, 1984; Enelow and Hinich, 1990] (the former is a standard yardstick for election studies and the latter receive increased attention in the literature [Elkind et al., 2017; Faliszewski et al., 2017a; Celis et al., 2018]):

1. For a probability value $p$, under the $p$-Impartial Culture model $(p-I C)$ each voter $i$ approves each candidate $c_{j}$ independently, with probability $p$.

2. In the Euclidean model, each candidate and each voter is associated with some point in a given $t$-dimensional Euclidean space $\mathbb{R}^{t}$ (typically $t \in\{1,2\}$ ). Each voter approves all the candidates whose points are within a given radius of the voter's point. In the $r$-Uniform Interval $(r-U I)$ and $r$-Uniform Square $(r$-US) models, the candidate and voter points are chosen uniformly at random from the interval $[0,1]$ or from the square $[0,1] \times[0,1]$, respectively, and each voter approves candidates within radius $r$.

Unless specified otherwise, our elections have $m=100$ candidates, $n=100$ voters, and committees of size $k=10$. Similar values are used in a number of papers, including those cited above. Nonetheless, we note that our parameters are somewhat special in that $n / k=k$; we checked several other election sizes and our results were qualitatively the same.

\section{Counting JR, PJR and EJR Committees}

We start our investigations by asking for the number of committees that provide the three variants of justified representation for a given election. We consider the following problems.

Definition 2. In the \#JR COMMITTEES problem we are given an election $E$ and an integer $k$, and we ask how many size- $k$ committees provide justified representation for $E$. The problems \#PJR COMMITTEES and \#EJR COMMITTEES are defined analogously, but for PJR and EJR, respectively.

These problems are interesting because knowing the number of JR, PJR, and EJR committees would give us a first hint on how restrictive these notions are. Unfortunately, the problems are \#P-hard and, thus, are unlikely to have polynomialtime algorithms (the class \#P is analogous to the class NP, but for the counting problems instead of the decision ones; see, e.g., the book of Hemaspaandra and Ogihara [2002]).

Theorem 1. \#JR COMmitTees, \#PJR CommitTeEs, and \#EJR COMMITTEES are \#P-hard.

Proof. We focus on \#JR Committees. We give a reduction from the \#P-hard \#PERFECT MATCHINGS problem [Valiant, 1979]. In this problem we are given a bipartite graph and the goal is to output the number of perfect matchings (i.e., of edge sets that cover every vertex exactly once). We describe a polynomial-time algorithm that takes an instance $I_{\mathrm{M}}$ of \#PERFECT MATCHINGS and transforms it into an instance $I_{\mathrm{J}}$ of \#JR COMMITTEES such that the number of JR committees in $I_{\mathrm{J}}$ equals the number of matchings in $I_{\mathrm{M}}$ plus some linear-time computable number depending only on $I_{\mathrm{M}}$.

Let $G$ be the bipartite graph of the \#PERFECT MATCHINGS instance. We assume w.l.o.g. that $G$ has an even number of vertices and set $2 r:=|V(G)|, r>1$. We construct the \#JR COMMITTEES instance $I_{\mathrm{J}}$ as follows. For each vertex $u \in V(G)$ we create a vertex voter $v(u)$. For each edge $e=\left\{u, u^{\prime}\right\}$ of $G$ we create an edge candidate $c(e)$ that is approved by $v(u)$ and $v\left(u^{\prime}\right)$. Additionally, we create two special voters $v_{1}^{*}$ and $v_{2}^{*}$ as well as a special candidate $c^{*}$ such that $c^{*}$ is approved by every (vertex and special) voter. Hence, for all $u \in V(G)$ we have $A_{v(u)}=\{c(e) \mid u \in e\} \cup\left\{c^{*}\right\}$ and for all $i \in\{1,2\}$ we have $A_{v_{i}^{*}}=\left\{c^{*}\right\}$. We set the committee size $k:=r$.

Let $x:=\left(\begin{array}{c}|E(G)| \\ r-1\end{array}\right)$ and let $y$ denote the number of perfect matchings in $G$. For the correctness of the reduction, we will now argue that $I_{\mathrm{J}}$ has exactly $x+y$ JR committees. Let us first analyze which JR committees are possible in the instance $I_{\mathrm{J}}$. Clearly, whenever the special candidate $c^{*}$ is part of the committee, then this committee provides justified representation because every voter approves $c^{*}$. In total, there are $x$ committees that include $c^{*}$ (we can fill up the committee with any set of $r-1$ further candidates). There may, however, be further JR committees that do not include $c^{*}$. We show that every such JR committee corresponds to a perfect matching in $G$ and vice versa, which finishes the proof.

First, assume that there is a perfect matching $M \subseteq E(G)$. Then, $W(M):=\{c(e) \mid e \in M\}$ is a JR committee of size $k$ because every vertex voter is covered by $W(M)$. Moreover, the two remaining special voters $v_{1}^{*}$ and $v_{2}^{*}$ cannot form a 1large, 1-cohesive group because $n / k=2 r+2 / r=2+2 / r>2$.

Second, assume that there is a size- $k \mathrm{JR}$ committee $W$ in $I_{\mathrm{J}}$ that does not include $c^{*}$. Then, $M(W):=\{e \mid c(e) \in W\}$ is a perfect matching. Assume towards a contradiction that some vertex $u$ from $G$ is not covered by any edge from $M(W)$. Then, since $c^{*}$ is not in $W$, the two special voters together with $v(u)$ form a 1-large, 1-cohesive group whose members do not approve any member of $W$. This contradicts the assumption that $W$ is a JR committee. It remains to show that no vertex is covered twice by $M(W)$. To see this, observe that $M(W)$ contains $r$ edges each covering two vertices and there are $2 r$ vertices in total. Thus, no vertex can be covered twice and $M(W)$ must be a perfect matching.

\section{Experimental Evaluation}

To get an idea regarding how many committees provide JR, PJR, and EJR for elections generated according to the $p$-IC, $r$-UI, and $r$-US elections, we performed the following experiment. We considered the following parameter values:

1. $p$ between 0.015 and 0.375 with step 0.15 for $p$-IC (which gives an average number of approved candidates 
Proceedings of the Twenty-Eighth International Joint Conference on Artificial Intelligence (IJCAI-19)

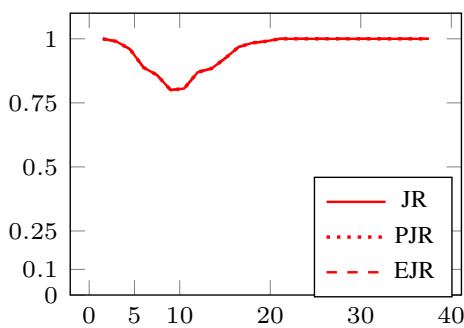

(a) $p$-Impartial Culture

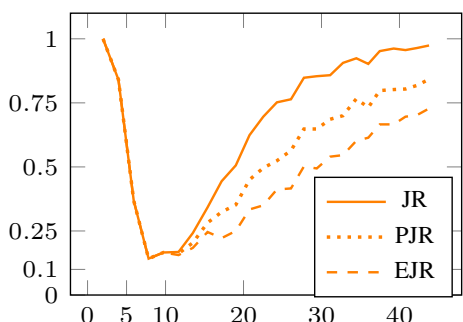

(b) $r$-Uniform Interval

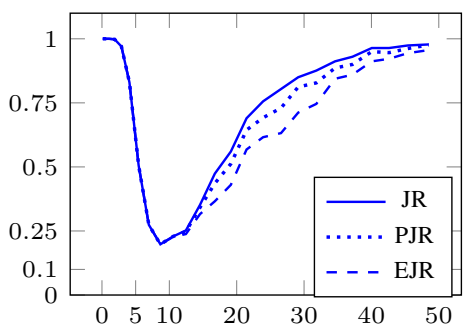

(c) $r$-Uniform Square

Figure 1: The probability that a randomly selected committee of size 10 provides JR, PJR, and EJR, for elections generated according to the $p$-IC model, the $r$-UI model, and $r$-US model, depending on the average number of candidates approved by a voter (on the $x$-axis).

per voter between 1.5 and 37.5$)$,

2. $r$ between 0.01 and 0.25 with step 0.01 for $r$-UI (which gives an average number of approved candidates per voter between 2 and 43), and

3. $r$ between 0.02 and 0.5 with step 0.02 for $r$-US (which gives an average number of approved candidates per voter between 0.12 and 48 ).

For each model of random elections and each parameter value, we generated 500 elections with 100 candidates and 100 voters each. For each of them we drew a single committee of size 10 uniformly at random and we checked if it provided JR, PJR, and EJR. We show our results in Figure 1. Instead of reporting on the $x$-axis the values $p$ and $r$ that we used, we give the average number of candidates approved by a single voter (this makes the results comparable between different distributions); the $y$-axis gives the probability that a randomly selected committee provides JR, PJR, and EJR. ${ }^{1}$

It is hardly surprising that when the average number of approved candidates is either very low or very high then nearly all random committees provide JR, PJR, and EJR (because either 1-large, 1-cohesive groups do not even appear in the profiles or each candidate covers a large fraction of $\ell$-large, $\ell$-cohesive groups for all values of $\ell$ ). It is, however, remarkable that for the whole range of the parameters there are many JR, PJR, and EJR committees. Indeed, under $p$-IC all the JR committees that we generated also provided PJR and EJR, and the probability that a random committee provides JR/PJR/EJR under $p$-IC does not fall below $80 \%$. For the case of $r$-UI and $r$-US models, up to about 10 approvals per voter, all the JR committees that we generated also provided PJR and EJR. For larger numbers of approved candidates there were JR committees that failed PJR and EJR. Yet, for the $r$ US model, such JR committees were not very frequent. For the case of $r$-UI the differences are more pronounced, but even in this model the probability of sampling a committee that satisfies each of JR, PJR, and EJR is high. (The lowest probability of generating such a committee for $r$-UI that we observed was $14.2 \%$; for $r$-US it was $19.8 \%$ ).

\footnotetext{
${ }^{1}$ While there is a fast algorithm for testing if a given committee provides JR, the same task for PJR and EJR is coNP-complete [Aziz et al., 2017; Aziz et al., 2018]. Thus, to check if a given committee does provide PJR or EJR, we formulated integer linear programs (ILPs) that test if a given committee fails these notions.
}

We also considered elections with 100 candidates, 200 voters and 200 candidates, 100 voters. The latter variant made a change shifting the minima towards 20 . Yet, the difference vanishes after normalizing the values on the $x$-axis wrt. the number of candidates. We omit details due to restricted space.

\section{JR Members and Justifying Groups}

One of the possible reasons why we have seen so many JR committees in the previous experiment is that, perhaps, for a committee to provide justified representation it suffices that it includes just a few key candidates, whereas all the other ones can be selected arbitrarily. We now check this hypothesis. We start by considering the notions of possible and necessary members of JR committees.

Definition 3. Let $E=(C, N)$ be an election and let $k$ be a committee size. We say that candidate $p \in C$ is a possible $J R$ member if there exists a size- $k$ committee that provides justified representation and that includes $p$. Candidate $p$ is a necessary JR member if he or she belongs to all size- $k$ committees that provide justified representation.

While checking if a candidate is a necessary JR member is intractable, many candidates belong to some JR committees.

Theorem 2. The problem of checking if a candidate is a necessary JR member for a given election is coNP-hard, and $\mathrm{co}-\mathrm{W}[1]$-hard when parameterized by the committee size $k$. The same holds for PJR and EJR.

Theorem 3. For each election $E=(C, N)$ and each committee size $k$, every candidate from $C$ that is approved by at least one voter belongs to at least one JR committee. The same holds for PJR and EJR.

Theorem 3 implies that for every election where each candidate is approved by at least one voter we have at least $m / k$ different committees that provide justified representation. This is not enough to explain the number of JR committees that we have seen in the preceding section and to obtain stronger results, we consider groups of possible JR members.

Definition 4. A set $S$ of candidates is an $n / k$-justifying group for an election if for each group $N^{\prime}$ of at least $n / k$ voters that approve at least one common candidate, at least one of these voters approves a member of $S$. 


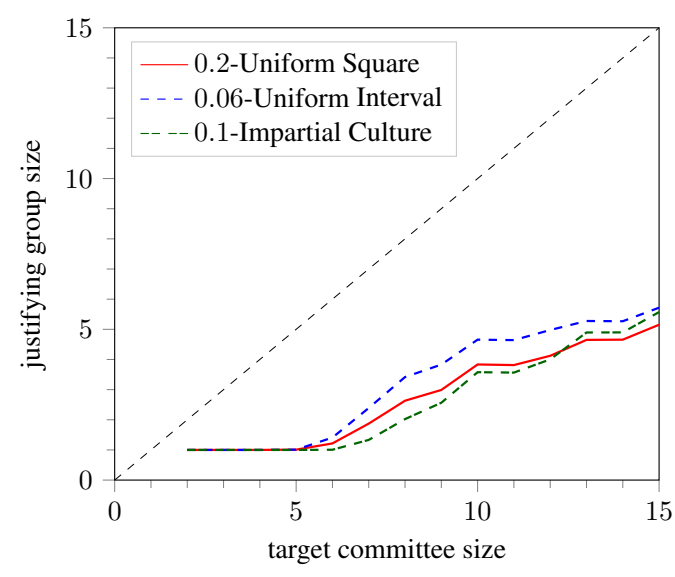

Figure 2: Average sizes of the $n / k$-justifying groups for our elections, depending on $k$.

Naturally, given an election with $n$ voters, each size- $k$ committee that provides justified representation is an $n / k$ justifying group for $E$ and, so, there always exists an $n / k$ justifying group of size $k$. However, it is far more interesting to ask for the size of the smallest such group. Indeed, if it turned out that such groups were significantly smaller than $k$, then it would explain why so many random committees provided justified representation. Unfortunately, computing the size of the smallest $n / k$-justifying group is intractable (the same holds for the notion of an $n / k$-justifying group adapted to the cases of PJR and EJR).

Theorem 4. The problem of deciding if there is an $n / k$ justifying group of at most a given size (for a given election and committee size) is NP-hard, and $\mathrm{W}[1]$-hard when parameterized by the committee size.

To deal with this hardness result, we express the problem as an ILP and solve it using Gurobi.

\section{Experimental Evaluation}

We consider elections with 100 candidates and 100 voters, for the 0.1-IC, 0.06-UI, and 0.2-US election models (these parameters are chosen so that, on the average, each voter approves about 10 candidates; we chose this value as we use the same distribution in the next experiment, where we focus on committee size 10 and it is very natural for a voter to approve about the same number of candidates as the size of the desired committee; other parameters would make sense as well). For $k \in\{2, \ldots, 15\}$, we computed the average sizes of the smallest $n / k$-justifying groups for the elections generated according to these models (1000 elections for each value of $k$ and each model). We present the results in Figure 2. As we can see, the justifying groups are typically quite small, which explains why we saw so many JR committees in the previous section. In the next section we will explore how much voter satisfaction/coverage we can achieve given so many degrees of freedom in JR committees.

It is natural to wonder if analogous results would hold for PJR and EJR, in scenarios where these notions differ from JR. Given that in all our experiments from the previous section a relatively high number of randomly selected committees satisfied PJR and EJR, we speculate that indeed this is the case.

\section{Voter Satisfaction Versus Coverage}

The AV rule outputs committees that provide the highest possible voter satisfaction, whereas the $\mathrm{CC}$ committees provide the highest possible voter coverage. Since CC satisfies JR but AV does not [Aziz et al., 2017], we know that there are JR committees that achieve the highest coverage values, but for some elections there are no JR committees with the highest possible voter satisfaction. In this section we ask what values of satisfaction/coverage are possible to achieve with JR committees (our work is inspired by Lackner and Skowron [2019], who focused on these values for prominent voting rules, mostly providing theoretical guarantees).

Definition 5. In the MAX SATISFACTION (COVERAGE) JR COMMITTEE problem we are given an election $E$, a committee size $k$, and an integer $x$, and we ask if there is a size- $k$ committee $W$ that provides justified representation and has score $_{\mathrm{AV}}(W) \geq x\left(\operatorname{score}_{\mathrm{CC}}(W) \geq x\right)$.

The Min Satisfaction (Coverage) JR Committee problems are defined analogously, but we ask if there is a JR committee that achieves at most a given value of voter satisfaction (voter coverage). We note that MAX COVERAGE JR COMMITTEE is equivalent to the WINNER DETERMINATION problem for the CC rule [Betzler et al., 2013]. All these problems are computationally intractable (also for the case of PJR and EJR) and we solve their JR variants using ILPs.

Theorem 5. Min SATisfaction (COVERAGE) JR COMMITTEE and MAX SATISFACTION (COVERAGE) JR COMMITTEE are all NP-hard, and $\mathrm{W}[1]$-hard when parameterized by the committee size $k$.

\section{Experimental Evaluation}

We have generated three elections ${ }^{2} E^{\mathrm{IC}}, E^{\mathrm{UI}}$, and $E^{\mathrm{US}}$, using the 0.1 -IC, 0.06 -UI, and 0.2-US models, with 100 candidates, 100 voters (their parameters are such that on average, each voter approves about 10 candidates). For each of the elections and for each pair of values $s \in\{0,1,2, \ldots, n m\}$ and $t \in[n]$, we computed if there exists (a) any committee that achieves voter satisfaction exactly $s$ and voter coverage exactly $t$, and (b) a JR committee that achieves these values. We also computed winning committees according to the AV, $\mathrm{CC}, \mathrm{PAV}$, and sequential Phragmén rules (this last rule is described, e.g., by Brill et al. [2017a]).

We present our results in Figure 3. It consists of two rows of plots. The top one should be read as follows. The $x$ axis of each of the plots corresponds to voter satisfaction values, and the $y$-axis corresponds to voter coverage values. If there is a dark red point on a given position $(s, t)$, then it means that there is a JR committee that achieves voter satisfaction $s$ and voter coverage $t$. If there is a light pink point, then it means that there is no JR committee with given parameters, but a non-JR committee with them exists. For AV,

\footnotetext{
${ }^{2}$ We repeated our experiment 60 times (20 times for the presented parameter settings and 40 times for slightly different ones, to check their robustness) and for all elections that we generated, we obtained results that were qualitatively the same.
} 

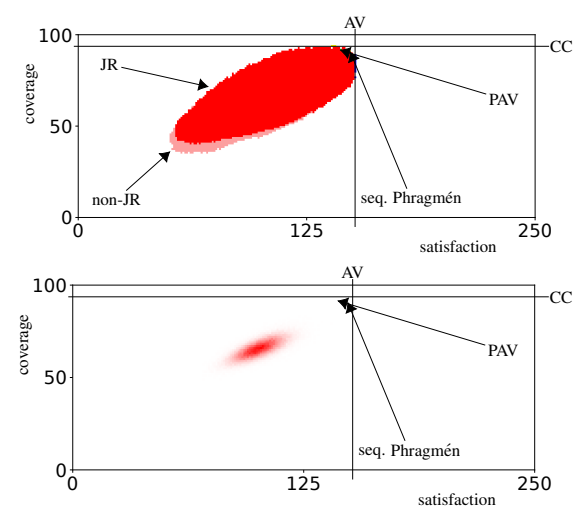

(a) 0.1-Impartial Culture, $\boldsymbol{E}^{\mathrm{IC}}$
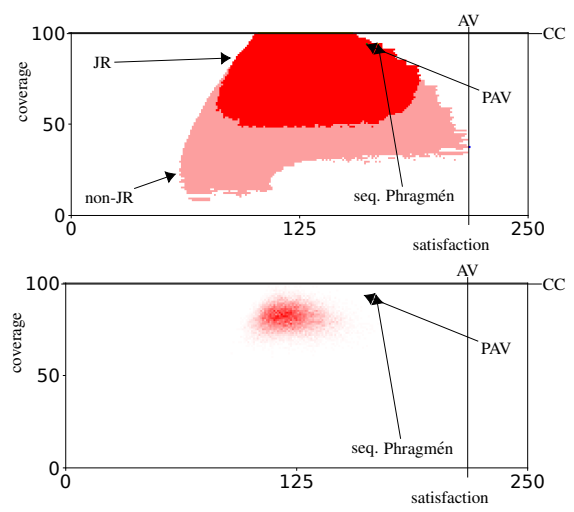

(b) 0.06-Uniform Interval, $\boldsymbol{E}^{\mathrm{UI}}$
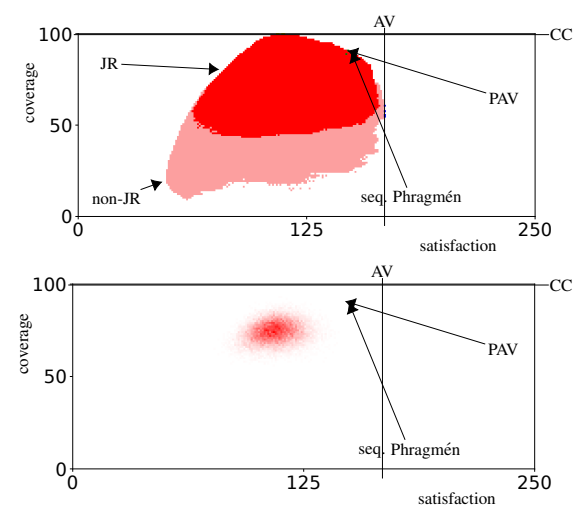

(c) 0.2-Uniform Square, $\boldsymbol{E}^{\mathrm{US}}$

Figure 3: Satisfaction vs. coverage for elections $\boldsymbol{E}^{\mathrm{IC}}, \boldsymbol{E}^{\mathrm{UI}}$, and $\boldsymbol{E}^{\mathrm{US}}$. In the top row, dark red points at coordinates $(s, t)$ indicate that there is a JR committee with voter satisfaction $s$, and with voter coverage $t$. Light pink points indicate that committees achieving a particular value exist, but there is no JR committee for it. In the bottom row, the darker the $(s, t)$ point is, the more JR committees (out of $10^{5}$ generated for each election) with satisfaction $s$ and coverage $t$ were generated.

a straight vertical line shows the satisfaction value achieved by the AV committees (whenever it touches the colored area, this indicates an AV committee that achieves a given satisfaction value). For CC we have an analogous horizontal line. For PAV and sequential Phragmén, we have arrows showing the $(s, t)$ points for which there is a PAV (resp. sequential Phragmén) committee (for these rules, we computed several committees and they were in the same area).

In the bottom row in Figure 3, we report results of the following experiment. For each of our three elections we have generated $10^{5}$ committees (of size 10 each), for each we have checked if it provided justified representation, and for those that did, we computed their satisfaction and coverage values. The plots show histograms, where the darker a given $(s, t)$ point is, the more JR committees with satisfaction $s$ and coverage $t$ our random process generated.

Our experiments confirm that JR committees are varied and can provide both high and low satisfaction and coverage values. Interestingly, the JR committees provided by our voting rules tend to give high satisfaction (resp. coverage) values and, indeed, are nearly on the satisfaction vs. coverage Pareto frontier. Yet, randomly generated JR committees are at some distance from the optimal values.

We repeated our experiments for the settings where each voter approved, on the average, between 23 and 26 candidates. In the experiments in Section 3 the ratio of sampled committees that satisfied EJR to those that satisfied JR was the lowest for such parameters (for the $r$-Uniform Interval and $r$-Uniform Square elections; for the $p$-Impartial Culture ones all JR committees also provided EJR). The nature of the results that we obtained was very similar to those presented in Figure 3 and, in particular, we still obtained large areas with JR committees and the randomly selected committees were focused in their specific subareas. Since many of the randomly selected JR committees also satisfy PJR/EJR, and since there are PJR/EJR committees located relatively far from the randomly sampled ones (e.g., those of PAV), we suspect that the areas of PJR/EJR committees are quite large, too.

\section{Discussion and Conclusions}

The understanding of the JR, PJR, and EJR notions that we get from our experimental study is quite different from the one based purely on theoretical results. In particular, we have shown settings where even satisfying EJR is quite easy, and we have shown that in these settings the number of committees providing JR, PJR, and EJR is rather large. Our results also reinforce the view that if one seeks a good representative committee, then it is not enough to simply require that the committee satisfies JR, PJR, or EJR, and one needs to put forward some additional criteria. Otherwise, there are too many, too varied committees to choose from.

We believe that one of the most interesting open problems arising from our work is a quest for a convincing model of generating elections where the notions of JR, PJR, and EJR differ significantly (in particular, where $\ell$-large, $\ell$-cohesive groups for different values of $\ell$ arise naturally). This was not the case for any model we analyzed and there seem to be no such models (trying to capturing reality) known in the literature. While it is clear that impartial culture does not capture reality either-and we use it as a yardstick because it is one of the most standard models - the Euclidean models seem to be more appealing and were used in several recent papers on multiwinner elections [Elkind et al., 2017; Faliszewski et al., 2017a; Celis et al., 2018]. We have tried different election types (not reported in the paper): the Polya-Eggenberger urn model [Berg, 1985], Euclidean elections with different distributions of the candidate and voter points, and various real-life elections from PrefLib [Mattei and Walsh, 2013]. We got qualitatively similar results.

\section{Acknowledgements}

We thank IJCAI reviewers for their useful comments. AK was supported by the DFG project "AFFA" (BR 5207/1 and NI369/15). PF was supported by the Friedrich Wilhelm Bessel Award from the Alexander von Humboldt Foundation when visiting TU Berlin. 


\section{References}

[Aziz et al., 2015] Haris Aziz, Serge Gaspers, Joachim Gudmundsson, Simon Mackenzie, Nicholas Mattei, and Toby Walsh. Computational aspects of multi-winner approval voting. In Proc. of AAMAS '15, pages 107-115, 2015.

[Aziz et al., 2017] Haris Aziz, Markus Brill, Vincent Conitzer, Edith Elkind, Rupert Freeman, and Toby Walsh. Justified representation in approval-based committee voting. Social Choice and Welfare, 48(2):461-485, 2017.

[Aziz et al., 2018] Haris Aziz, Edith Elkind, Shenwei Huang, Martin Lackner, Luis Sánchez-Fernández, and Piotr Skowron. On the complexity of extended and proportional justified representation. In Proc. of AAAI' 18 , pages 902-909, 2018.

[Berg, 1985] Sven Berg. Paradox of voting under an urn model: The effect of homogeneity. Public Choice, 47(2):377-387, 1985.

[Betzler et al., 2013] Nadja Betzler, Arkadii Slinko, and Johannes Uhlmann. On the computation of fully proportional representation. Journal of Artificial Intelligence Research, 47:475-519, 2013.

[Brill et al., 2017a] Markus Brill, Rupert Freeman, Svante Janson, and Martin Lackner. Phragmén's voting methods and justified representation. In Proc. of AAAI'17, pages 406-413, 2017.

[Brill et al., 2017b] Markus Brill, Jean-François Laslier, and Piotr Skowron. Multiwinner approval rules as apportionment methods. In Proc. of AAAI'17, pages 414-420, 2017.

[Byrka et al., 2018] Jarosław Byrka, Piotr Skowron, and Krysztof Sornat. Proportional approval voting, harmonic k-median, and negative association. In Proc. of ICALP' 18 , pages 26:1-26:14, 2018.

[Celis et al., 2018] L. Elisa Celis, Lingxiao Huang, and Nisheeth Vishnoi. Multiwinner voting with fairness constraints. In Proc. of IJCAI' 18, pages 144-151, 2018.

[Chamberlin and Courant, 1983] John R. Chamberlin and Paul N. Courant. Representative deliberations and representative decisions: Proportional representation and the Borda rule. American Political Science Review, 77(3):718-733, 1983.

[Elkind et al., 2017] Edith Elkind, Piotr Faliszewski, JeanFrançois Laslier, Piotr Skowron, Arkadii Slinko, and Nimrod Talmon. What do multiwinner voting rules do? An experiment over the two-dimensional euclidean domain. In Proc. of AAAI '17, pages 494-501, 2017.

[Elkind, 2017] Edith Elkind. Justified Representation in Multiwinner Voting: Axioms and Algorithms. In Proc. of FSTTCS '17, volume 93 of LIPICs, pages 1:1-1:10. Schloss Dagstuhl-Leibniz-Zentrum fuer Informatik, 2017.

[Enelow and Hinich, 1984] James M. Enelow and Melvin J. Hinich. The Spatial Theory of Voting: An Introduction. Cambridge University Press, 1984.

[Enelow and Hinich, 1990] James M. Enelow and Melvin J. Hinich. Advances in the Spatial Theory of Voting. Cambridge University Press, 1990.
[Faliszewski et al., 2017a] Piotr Faliszewski, Piotr Skowron, Arkadii Slinko, and Nimrod Talmon. Multiwinner rules on paths from $k$-Borda to Chamberlin-Courant. In Proc. of ICJAI'17, pages 192-198, 2017.

[Faliszewski et al., 2017b] Piotr Faliszewski, Piotr Skowron, Arkadii Slinko, and Nimrod Talmon. Multiwinner voting: A new challenge for social choice theory. In U. Endriss, editor, Trends in Computational Social Choice, pages 27 47. AI Access Foundation, 2017.

[Hemaspaandra and Ogihara, 2002] Lane A. Hemaspaandra and Mitsunori Ogihara. The Complexity Theory Companion. Springer-Verlag, 2002.

[Kilgour, 2010] D. Marc Kilgour. Approval balloting for multi-winner elections. In Handbook on Approval Voting, pages 105-124. Springer, 2010. Chapter 6.

[Lackner and Skowron, 2018] Martin Lackner and Piotr Skowron. Consistent approval-based multi-winner rules. In Proc. of EC'18, pages 47-48, 2018.

[Lackner and Skowron, 2019] Martin Lackner and Piotr Skowron. A quantitative analysis of multi-winner rules. In Proc. of IJCAI' 19, 2019.

[Lu and Boutilier, 2011] Tyler Lu and Craig Boutilier. Budgeted social choice: From consensus to personalized decision making. In Proc. of IJCAI'11, pages 280-286, 2011.

[Mattei and Walsh, 2013] Nicholas Mattei and Toby Walsh. Preflib: A library for preferences. In Proceedings of the 3nd International Conference on Algorithmic Decision Theory, pages 259-270, 2013.

[Procaccia et al., 2008] Ariel D. Procaccia, Jeffrey S. Rosenschein, and Aviv Zohar. On the complexity of achieving proportional representation. Social Choice and Welfare, 30(3):353-362, 2008.

[Sánchez-Fernández et al., 2017] Luis Sánchez-Fernández, Edith Elkind, Martin Lackner, Norberto Fernández, Jesús Arias-Fisteus, Pablo Basanta-Val, and Piotr Skowron. Proportional justified representation. In Proc. of AAAI '17, pages 670-676, 2017.

[Skowron et al., 2015] Piotr Skowron, Piotr Faliszewski, and Arkadii Slinko. Achieving fully proportional representation: Approximability result. Artificial Intelligence, 222:67-103, 2015.

[Skowron et al., 2016] Piotr Skowron, Piotr Faliszewski, and Jérôme Lang. Finding a collective set of items: From proportional multirepresentation to group recommendation. Artificial Intelligence, 241:191-216, 2016.

[Skowron et al., 2017] Piotr Skowron, Martin Lackner, Markus Brill, Dominik Peters, and Edith Elkind. Proportional rankings. In Proc. of ICJAI' 17, pages 409-415, 2017.

[Thiele, 1895] Thorvald N. Thiele. Om flerfoldsvalg. In Oversigt over det Kongelige Danske Videnskabernes Selskabs Forhandlinger, pages 415-441. 1895.

[Valiant, 1979] Leslie G. Valiant. The complexity of enumeration and reliability problems. SIAM Journal on Computing, 8(3):410-421, 1979. 